COMPETITIVE EQUILIBRIUM FOR

INCOMPLETE MARKET STRUCTURES

Yves YOUNES

A. - General Market Structures and Demand Theory

October 19985

Revised June and October 1986

$N^{\circ} 8623$ 


\section{COMPETITIVE EQUILIBRIUM FOR INCOMPLETE MARKET STRUCTURES}

\section{A - General Market Structures and Demand Theory}

\section{A B $\quad$ S T T R A C T}

The objective is to analyze, in a natural way, problems of existence and determinacy of competitive equilibria when the markets structures are incomplete. Then, within the limits of the differential approach, a general formalism is proposed, which allows to study easily the problems at hand. The usual properties of demand functions (behaviour near the boundary, Slutsky matrice and Walras Law) are carried over to the case the typical consumer chooses his net trade no more necessarily in an hyperplane, but more generally in a vector subspace of any dimension. These results point out the fact that the notion of a price vector has to be replaced by the notion of a price system or, alternatively by the notion of a vector of exchange ratios between bundles of commodities.

Journal of Economic Literature 020
Key words :
$\begin{aligned} \text { - } & \text { - } \text {-enmoral Market bundles } \\ & \text { - Exchange Ratio } \\ & \text { - Price System } \\ & \text {-Demand Function }\end{aligned}$

\section{EQUILIBRE CONCURRENTIEL ET STRUCTURES DE MARCHE INCOMPLETES}

A - Structures Générales de marché et théorie de la demande

$$
\text { R É S U'M É }
$$

L'objectif de l'étude est d'examiner, d'une manière naturelle, les problèmes d'existence et de nombre d'équilibres concurrentiels quand la structure de marché est incomplète. Cette analyse est faite dans les limites (habituellement retenues) de 1 'hypothèse de différentiabilité. Alors, un formalisme général est proposé qui permet de manier aisément les problèmes envisagés. Les propriétés habituelles des fonctions de demande (comportement aux limites, matrice de Slutsky et loi de Walras) sont généralisées au cas où le consommateur typique choisit son échange net non plus nécessairement dans un hyperplan, mais dans un sous-espace vectoriel de dimension quelconque. Les résultats obtenus montrent que la notion fondamentale est celle de système de vecteurs prix (ou de rapports d'échange entre paniers de biens) et non plus de vecteur prix.

- Paniers de biens

Mots clefs : - Structures générales de marché

- Rapport d'échange

- Système de prix

- Fonction de demande 


\title{
Competitive Equilibrium for Incomplete Market Structures
}

\author{
Y. Younès
}

\section{A. General Market Structures and Demand Theory. October 1985 \\ Revised June and October 1986}

Recently, the theory of competitive equilibrium in an incomplete market setting was given considerable attention. After Radner's pathbreaking contribution (1972), 0. Hart (1975) provided a simple example of inexistence of a competitive equilibrium due to some discontinuity of the demand function. This lack of continuity of the demand function is due to the fact that when the price vector varies, the subspaces (no more hyperplanes necessarily) over which the typical consumer maximizes utility can have different dimensions. In order to overcome this difficulty, some authors restricted their analysis to the case where, whatever is the price vector, the subspaces have always the same dimension. For the case of financial securities, see J. Werner (1985), D. Cass (1984) and D. Duffie (1985). For the case of securities denominated in terms of goods, see S. Chae (1985). On the contrary, other authors' contributions bear on the generic existence of a competitive equilibrium. When the structure is nearly complete, using the notion of Walras Equilibria, it is possible to get generic existence as in D. MacManus (1984), R. Repullo (1983) and M. Magill and W. Shafer (1985). When the market structure is not nearly complete, the notion of Walrasian Equilibrium cannot any more be used as a benchmark. Following an Idea contained in M. Magill and W. Shafer (1985), D. Duffle and W. Shafer (1985) use the notion of pseudo-equilibrium (along with the notion of the manifold of all subspaces of dimension $n$ of $\mathbb{R}^{r}$ ) in order to get generic existence of an equilibrium. It is also the point of view adopted by H. Polemarchakis (1986). Along with the question of existence of an equilibrium, the problem 
of determinacy of equilibrium have recelved some attention. Although it was proved that if the return of securities are denominated in goods, the equilibrium is not really more indeterminate than in the case of a complete set of markets, it was shown that there is real indeterminacy when assets are financial ones (Y. Balasko and D. Cass (1986), J. Geanakoplos and A. MasColell (1985) and H. Polemarchakis (1986)).

The modest objective of this paper is to try to build a theory of competitive equilibrium for incomplete market structures which is roughly similar to the one which is valid when the set of markets is complete, given that the differential viewpoint is accepted. For that, one tries first to define a market structure in a general way, i.e. as a set of activities of exchanges. Given the differential viewpoint, it seems fairly general and covers the case of spot markets with financial and/or real securities. Then one tries to construct a theory of demand when each consumer maximizes utility over a subspace $z$ of dimension $n$, without defining the set of market structures which gives rise to this set of subspaces. One gets a straightforward generalization of the usual (demand) theory about the individual demand functions $\xi_{i}\left(z, w_{i}\right)^{(1)}$ and aggregate demand function $\sum \xi_{i}\left(Z, w_{1}\right)$ (behavior of these functions near the boundary, Slutsky Matrix and Walras law): These results which are gathered in a first chpater, points out to the fact that the notion of a price vector has to be replaced by the notion of a price system (alternatively by the notion of a vector of exchange ratios between bundles of commodities) in order to study existence and determinacy in a natural way, at least within the limits of the differential approach.

For studying generic existence and determinacy of a competitive equilibrium for general market structures, we proceed in the following way. It turns out that $\sum_{i} \xi_{i}\left(Z, w_{i}\right)=0$ gives rise to a smooth manifold. Then one 
studies the classes of markets structures which, when palred with a subset of Z's generates a manifold. Existence and determinacy are then studied through the analysis of the intersection of the manifold induced by $\sum \xi_{i}\left(z, w_{i}\right)=0$ and one of these manifolds. For instance with each market structure with "financial securities", it is possible to associate a whole class of market structures with "real securities".

These last results are presented in the second chapter.

I.

Consider a set of exchange economies with $r$ goods $h$ and $m$ consumers $i$. Each consumer $i$ is endowed with a utility function $\tilde{u}_{i}$ defined on $x_{i} \equiv \mathbb{R}_{++}^{r}$ and initial endowments $w_{i} \in \mathbb{R}_{++}^{r}$. One makes the following restrictive assumption:

Al: For each $1, \tilde{u}_{1}$ is differentiably strictly increasing and strictly quasi concave. The closure of the indifference hypersurfaces is contained in $\mathbb{R}_{++}^{\mathrm{r}}$. Finally $w_{i} \in \mathbb{R}_{++}^{\mathrm{r}}$.

Remark: Let $u_{i}$ be the the Hessian of $\tilde{u}_{i}$. Then Al implies that $z^{\prime} U_{i} z<0$ for every $z$ belonging to the set $\left\{z \in \mathbb{R}^{x} \mid u^{\prime} z=0, z \neq 0\right\}$ where $u$ is the gradient of $\tilde{u}$.

A bundle of goods is a point $\lambda$ in $\mathbb{R}_{+}^{\mathfrak{r}} \backslash\{0\}$. Define a simple market as the description of exchange between two bundles of goods. There are $n$ simple markets $\alpha$. Denote by $\lambda^{\alpha+}$ the first bundle of goods and by $\lambda^{\alpha-}$ the second one, on market $\alpha$. Formally, one assumes:

A2: For each $\alpha: \lambda_{h}^{\alpha+} \cdot \lambda_{h}^{\alpha-}=0$ for each $h$. For each $\alpha, \lambda^{\alpha+} \in \mathbb{R}_{+}^{r} \backslash\{0\}$ and $\lambda^{\alpha-} \in \mathbb{R}_{+}^{r} \backslash\{0\}$. 
A2 means that the same good cannot enter in both bundles which are exchanged on the simple market $\alpha$. A (simple) market structure can then be defined as a couple of matrices $\Lambda=\left\{\Lambda^{+}, \Lambda^{-}\right\}$where $\Lambda^{+}$and $\Lambda^{-}$are $r \times n$ nonnegative matrices. The couple constructed by the column $\alpha$ of $\Lambda^{+}\left(\lambda^{\alpha+}\right)$ and the column $\alpha$ of $\Lambda^{-}\left(\lambda^{\alpha-}\right)$ refers to the two bundles of goods which are exchanged on market $\alpha$.

Let be $\pi^{\alpha}$ the exchange ratio at which the two bundles are exchanged on the (simple) market $\alpha$. Define the matrix $\tilde{T}(\pi)$ by

$$
\tilde{T}(\pi)=\Lambda^{+}-\Lambda^{-}[\pi]
$$

where $[\pi]$ is the $n \times n$ diagonal matrix with the $\pi^{\alpha} s$ on the diagonal. The following assumption is made only for the sake of simplicity.

A.3: One has $n \leq r-1$, i.e. the number of simple markets is strictly smaller than the number of goods.

Let be $z_{i} \in \mathbb{R}^{r}$ consumer $i^{\prime}$ 's net trade. When $\pi \in \mathbb{R}_{++}^{r}$ is given, $\tilde{T}(\pi)$ defines the activities of exchange that consumer 1 faces. Let be $a_{i}^{a}$ the intensity of use of the activity of exchange $\alpha$ by agent $i$. Then:

$$
z_{1}=\tilde{T}(\pi) a_{i}
$$

where $a_{i}=\left\{a_{1}^{1}, \ldots, a_{1}^{\alpha}, \ldots, a_{1}^{n_{1}}\right\}$.

Remark: Given Al, our construction is more general than it appears. Define more generally a market $\beta$ as a vector subspace $z^{\beta}$ of $\mathbb{R}^{r}$ (See D. Gale (1982)). On market $\beta, b(B)$ disjunct bundles of goods are exchanged. If Al 
is made all allocations are interior ones and marginal rates of substitution, which are strictly positive, vary continuously with allocations. Thus with each market $\beta$, one can associate $b(\beta)-1$ simple markets $\alpha$ : on each simple market $\alpha$, each of the first $[b(\beta)-1]$ bundles is exchanged for the last one. (2) Finally, the following assumption means that all goods can be traded.

A4: Th, there exists $\alpha$ such that $\lambda_{h}^{\alpha} \neq 0$.

Remark: A2-A4 imply that, whatever is $\pi \in \mathbb{R}_{++}^{r}$, it is possible to trade every good $h$.

Given $\Lambda$, one can associate with each $p \in \mathbb{R}_{++}^{r}$, one $\pi \in \mathbb{R}_{++}^{\mathrm{n}}$ by the function $F_{\Lambda}: \mathbb{R}_{++}^{r}+\mathbb{R}_{++}^{n}$ defined as follows:

$$
\forall \alpha: \pi^{\alpha}=\sum_{h} \lambda_{h}^{\alpha+} p_{h} / \sum_{h}^{\sum} \lambda_{h}^{\alpha-} p_{h}
$$

one defines also $T(p) \equiv \tilde{T}\left(F_{\Lambda}(p)\right)$.

Each $\pi \in F_{\Lambda}\left(\mathbb{R}_{++}^{r}\right)$ is sald to satisfy the no arbitrage condition for $\Lambda$. In order to show the relevance of our construction when Al is made, let us consider a canonical example.

Example 1: Our formalism covers the case of a market structure with spot markets and (Radner-Hart) securities (S.M.S.). There are two periods (period 0 and period 1) and $\sigma$ states $s$ at period 1 . There are $r$ goods in each state $s \geq 1$. Thus $\mathbf{r}=\left(\sigma \mathbf{r}^{\prime}+1\right)$. There are $k$ securities $j$. The price of security $j$ is $\pi^{j}$ (in terms of the sole good of period 0 ) and it gives as returns the bundle of goods $b^{j} \in \mathbb{R}_{+}^{\sigma r^{+}}$. With this market structure is 
associated the following $\tilde{\mathrm{T}}(\pi)$

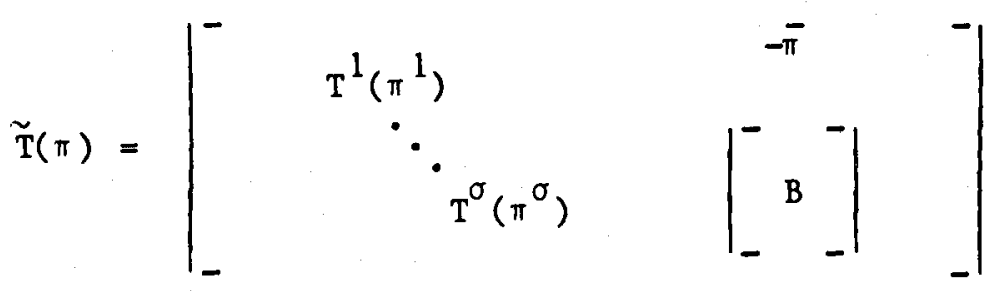

where for $s=1, \ldots, \sigma$

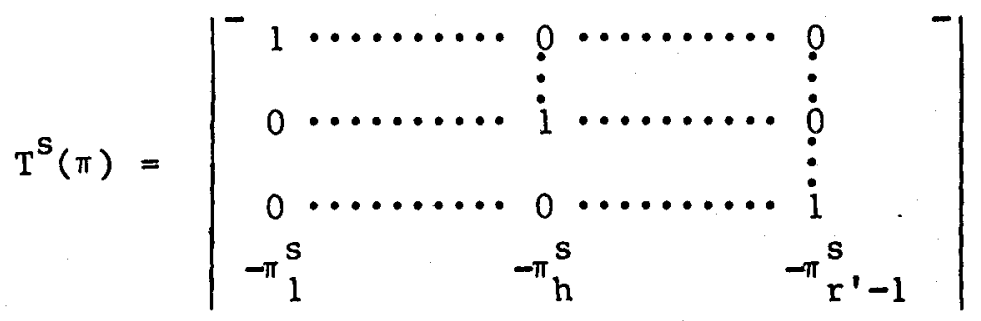

is a $r^{\prime} \times\left(r^{\prime}-1\right)$ matrix, $\bar{\pi}$ is a $k$ row-vector placed at the row of the good of period 0 (it is the $k$-vector of prices of securities in terms of the good of period 0$)$ and $B$ is $a\left(r^{\prime} \times \sigma\right) \times k$ non negative matrix of securities returns. It appears immediately that the matrix gotten by taking the first $\sigma\left(r^{\prime}-1\right)$ columns plus one of the last $k$ ones has rank $\sigma\left(r^{\prime}-1\right)+1$ whatever is $\pi$. It appears also that if $B$ has rank $k$ which implies $k \leq \sigma-$ and if for each $s \geq 1$, there is only one good $h(s)$ for which the corresponding row of $B$ is not zero, then $\tilde{T}(\pi)$ has constantly rank $n$, whatever is $\pi$, where $n=\sigma\left(r^{\prime}-1\right)+k \leq r-1$. (See e.g., S. Chae (1985)) .

\section{II}

The objective of this part is to define the equilibrium concept. Let us assume that $\Lambda$ is given. A net trade $z_{1}$ is possible for $i$ iff (i) th: $w_{i h}+z_{i h}>0$ 
(ii) $\exists \pi_{i} \in \mathbb{R}_{+}^{\mathrm{n}}$ such that $z_{i} \in z_{i}$ where $z_{i}$ is the subspace generated by $\tilde{T}\left(\pi_{1}\right)$.

Write $a=\left[a^{1}, \ldots, a^{\alpha}, \ldots, a^{n}\right]$.

Remark: $z_{1}=\left\{z \in \mathbb{R}^{r} \mid \exists a \in \mathbb{R}^{n}\right.$ such that $\left.\tilde{T}\left(\pi_{1}\right) a=z\right\}$. Thus (i1) of possibility can read: $\exists a_{i}$ and $\pi_{i}$ such that $\tilde{T}\left(\pi_{i}\right) a_{i}=z_{i}$. $z=\left[z_{1}, \ldots, z_{m}\right]$ is feasible if for each $1, z_{i}$ is possible and for each $\alpha: \sum t^{\alpha}\left(\pi_{1}^{\alpha}\right) a_{i}^{\alpha}=0$ (balance). Obviously, one is interested in the case where all consumers trade at the same exchange ratio for each $\alpha$. Thus we shall require $\pi_{i}=\pi \psi_{i}$. In this case $\sum t^{\alpha}\left(\pi^{\alpha}\right) a_{i}^{\alpha}=0 \forall \alpha$ is implied by $\sum a_{i}=0$. Given Al, the vector $\pi=\left[\pi^{1}, \ldots, \pi^{\alpha}, \ldots, \pi^{n}\right]$ satisfies the no arbitrage condition if there exists $p \in \stackrel{\circ r-1}{s}$ such that

$$
p^{\prime} \cdot \tilde{T}(\pi)=0
$$

Notice that given $\pi \in \mathbb{R}_{++}^{n}$, the set of $p \in \mathbb{R}^{\mathbb{r}}$ such that $p^{\prime} \tilde{T}(\pi)=0$ is a subspace of dimension $r-n$, at least.

Definition 1: Given $\Lambda$, an equilibrium is a triple $\left[\pi^{*}, z^{*}, z^{*}\right]$ such that

(i) $2^{*}$ is the subspace generated by $\tilde{\mathrm{T}}\left(\pi^{*}\right)$

(ii) $z^{*}\left[z_{1}^{*}, \ldots, z_{m}^{*}\right]$ if feasible with respect to $\Lambda$.

(iii) $\forall_{i}: z_{i}^{*}$ maximizes $\tilde{u}_{i}\left(w_{i}+z_{i}\right)$ over the set $z^{*} n x_{i}$

Notice that $\mathrm{Z}^{*}$ can be of dimenston smaller than $\mathrm{n}$ if the $\mathrm{n}$ vectors of $\tilde{\mathrm{T}}\left(\pi^{*}\right)$ happen to be not linearly independent.

It is possible to characterize an equilibrium in two (dual) ways. 
First characterization: choose a basis for the orthogonal complement of $z^{*}\left(z^{*}\right.$ can have dimension smaller than $\left.n\right) \quad P=\left\{p^{1}, \ldots, p^{j}, \ldots, p^{r-n}\right\}$ where $\Psi_{j}: p^{j}$ is a (column) vector of dimension $r$. Then

$$
\begin{aligned}
& \text { (i) } \sum z_{i}^{*}=0 \\
& \text { (ii) } \forall_{i}: \mathrm{P}^{\prime} z_{i}^{*}=0 \\
& \text { (iii) } \forall_{i}: u_{i}=\mathrm{Pb}_{i} \text { for some } b_{i} \in \mathbb{R}^{\mathrm{r}-\mathrm{n}} \text {. }
\end{aligned}
$$

Second characterization:

$$
\exists a=\left[a_{1}, \ldots, a_{m}\right] \text { with } a_{i} \in \mathbb{R}^{n}, \forall_{i} \text {, such that: }
$$

$$
\begin{aligned}
& \text { (1) } \sum_{i} a_{i}=0 \\
& \text { (ii) } \forall_{i}: \tilde{T}\left(\pi_{i}^{*}\right) a_{i}=z_{i}^{*} \\
& \text { (iii) } \Psi_{i}: u_{i}^{\prime} \tilde{T}\left(\pi^{*}\right)=0 .
\end{aligned}
$$

In the first characterization, $P$ is called a price system. Up to a multiplication by a regular $(r-n) \times(r-n)$ matrix, it is uniquely defined. Now as it is well known, when $\tilde{T}(\pi)$ has not a constant rank (let us say $n$ ) when $\pi$ varies in $\mathbb{R}^{\mathrm{n}}$, the demand function generated by (iii) of definition 1 is not necessarily continuous. Thus in order to tackle with existence problems, one introduces the notion of pseudo equilibrium.

Definition 2: Given $\Lambda$, a pseudo equilibrium is a triple $\left[\pi^{*}, z^{*}, z^{*}\right]$ such that

(i) $z^{*}$ is a subspace of dimension $n$ which contains the subspace generated by $\tilde{T}\left(\pi^{*}\right)$.

(ii) $\quad \sum z_{i}^{*}=0$

(iii) $\forall_{i}: z_{i}^{*}$ maximizes $\tilde{u}_{i}\left(w_{i}+z_{i}\right)$ over the set $z^{*} \cap x_{i}$. 
Let us say that $\Lambda$ is regular if for each $\pi \in \mathbb{R}_{++}^{\mathrm{n}}, \tilde{\mathrm{T}}(\pi)$ has rank $\mathrm{n}$ and that $\Lambda$ is quasi-regular if there exists $p \in \mathbb{R}_{++}^{\mathbf{r}}$ such that $\tilde{T}[F(p)]$ has rank $n . \Lambda^{1}$ and $\Lambda^{2}$ use strongly equivalent if for every $\left[w,\left(\tilde{u}_{i}\right)_{1}\right]$, the set of equilibria with respect to $\Lambda^{2}$ is equal to the set of equilibria with respect to $\Lambda^{1} \cdot \Lambda^{1}$ and $\Lambda^{2}$ are weakly equivalent if they are quasi regular and if for every $\left[w,\left(\tilde{u}_{i}\right)_{i}\right]$, the set of pseudo equilibria with respect to $\Lambda^{1}$ is equal to the set of pseudo equilibria with respect to $\Lambda^{2}$. It follows that all quasi-regular $\Lambda$ with $n=r-1$ simple markets are weakly equivalent because each one is weakly equivalent with the structure with a complete set of markets which gives rise to a Walrasian Equilibrium.

\section{III}

It is possible to endow the set of all $n$ dimensional subspaces with a differential structure. The reader is referred to J. Dieudonne (1970) (XVI11) and to D. Duffie and W. Shafer (1985)).

Let us note $G_{r, n}$ the set of all $n$-dimensional subspaces of $\mathbb{R}^{r}$. There are two (dual) ways of constructing an atlas on $G_{r, n}$. Notice that $G_{r, n}$ is a metric space. The first way is the following. Choose a $z \in G_{r, n}$, and a basis $T$, i.e. a $r \times n$ matrix of rank $n$ such that $z=\left\{z \in \mathbb{R}^{\mathbf{r}} \mid \exists a \in \mathbb{R}^{\mathfrak{n}}\right.$ such that $\left.\mathrm{T} a=z\right\}$. Clearly, if $T$ is a basis for $\mathrm{Z}, \mathrm{TC}$ is also a basis where $\mathrm{C}$ is a regular $\mathrm{n} \times \mathrm{n}$ matrix. By assumption, there are $n$ rows of $T-n=\left\{h_{1}>\ldots>h_{j}>\ldots>h_{n}\right\}$ - such that the submatrix formed with these $n$ rows is regular. In order to emphasize the fact that we are focusing on $\eta$, we write $\eta^{T}$. Clearly there is $c$ such that the square submatrix of $n^{T \cdot C}$ formed with the rows $\left.\left\{h_{1}>\ldots\right\rangle h_{n}\right\}$ is the unitary square matrix. In this case, one refers to a normalized basis. For each $\eta$, consider the class $\tau_{\eta}$ of all ${ }_{n} \mathrm{~T}$ which are normalized. One 
can identify it with $\mathbb{R}^{n(r-n)}$. For each $n$ (there is a finite number of $\left.\eta^{\prime} s\right)$ let $W_{\eta}$ be the set in $G_{r, n}$ defined by:

$W_{\eta}=\left\{z \in G_{r, n} \mid \exists_{\eta} T \in \eta \approx \mathbb{R}^{n(r-n)}\right.$ such that $\eta^{T}$ is a basis for $\left.z\right\}$.

Define also $\phi_{\eta}: w_{\eta} \rightarrow \mathbb{R}^{n(r-n)}$ by associating with each $z \in w_{\eta}$ the $n^{T} \in \mathbb{R}^{n(r-n)}$ which is a normalized basis for $z$. $\left\{w_{\eta}\right\}_{\eta}$ is then an open cover of $G_{r, n}$ and $\phi_{\eta}$ is, for each $n$, a homeomorphism of $w_{\eta}$ onto $\mathbb{R}^{n(r-n)}$. Then $\left\{w_{n}, \phi_{n}\right\}$ is an atlas on $G_{r, n}$, making it a compact smooth manifold of dimension $n(r-n)$.

We shall use here mainly a dual way of constructing an atlas on $G_{r, n}$. The idea is to define a subspace of dimension $n$ of $\mathbb{R}^{\mathfrak{r}}$ through its orthogonal complement of dimension $(r-n)$ in the same way as an hyperplane is defined through its orthogonal complement of dimension 1, 1.e. a price vector, in the usual consumer's theory.

Any $(r-n) \times r$ matrix $P$ which is of rank $(r-n)$ induces a $n-$ dimensional subspace of $\mathbb{R}^{r}$ by

$$
Z=\left\{z \in \mathbb{R}^{r} \mid P^{\prime} z=0\right\}
$$

One can refer to $P$ as a price system. Moreover if $C$ is a $(r-n) \times(r-n)$ regular matrix, then $P$ and $P \cdot C$ will induce the same $Z$. Thus one can identify $Z$ and the equivalent class of $P^{\prime} s$ which induce it. As $P$ has full rank, there are $(r-n)$ rows which are linearly independent. Let us choose rows $\left\{h_{1}>\ldots>h_{r-n}\right\}=\gamma$. In order to emphasize the fact that we are focusing on rows $\left\{h_{1}, \ldots, h_{r-n}\right\}$, one shall write $\gamma^{P}$. Clearly, there is $C$ such that the square submatrix of $\gamma^{P C}$ formed by the rows $\left\{h_{r}, \ldots, h_{r-n}\right\}$ is 
the unitary square matrix. In this case, one refers to a normalized $\gamma^{P}$. For each $\gamma$, consider the class $J_{\gamma}$ of all $\gamma^{P}$ which are normalized. Clearly, one can identify $J_{\gamma}$ with $\mathbb{R}(r-n)$. For each $\gamma$, define $W_{\gamma}=\left\{z \in G_{r, n} \mid \exists \gamma_{\gamma} P \in J_{\gamma} \approx \mathbb{R}^{n(r-n)}\right.$ such that $Z$ is induced by $\left.\gamma^{P}\right\}$. Define $\psi_{\gamma}: w_{\gamma}+\mathbb{I R}^{n(r-n)}$ by associating with each $z \in W_{\gamma}$ the ${ }_{\gamma} P$ which induces 1t. Then $\left\{W_{\gamma}\right\}_{\gamma}$ is an open cover of $G_{r, n}$ and $\psi_{\gamma}$ is, for each $Y$, a homeomorphism of $W_{\gamma}$ onto $\mathbb{R}^{n(n-r)}$. Then $\left\{W_{\gamma}, \psi_{\gamma}\right\}$ is an atlas on $G_{r, n}$, making it a compact smooth manifold of dimension $n \times(r-n)$.

\section{IV}

The objective of this part is to gather in a simple way some elements of the theory of consumer's behavior, when the typical consumer's net trade is constrained to belong to a subspace of dimension $n$ of $\mathbb{R}^{r}$, with $\mathrm{n} \leq \mathrm{r}-1$, and no more necessarily to an hyperplane 1.e. $\mathrm{n}=\mathrm{r}-1$. Let be $\tilde{G}_{r, n}$ the set of subspaces. $Z$ of dimension $n$ defined in the following way

$$
\tilde{G}_{r, n}=\left\{z \in G_{r, n} \mid \exists u \in \mathbb{R}_{++}^{r} \text { s.t. } u \in z^{\perp}\right\}
$$

where $z^{\perp}$ is the orthogonal complement of $z$ in $\mathbb{R}^{r}$. For each $z \in \tilde{G}_{r, n}$, the set defined for some $w \in \mathbb{R}_{++}^{r},\left\{z \in z \mid w+z \in \mathbb{R}_{++}^{r}\right\}$ is bounded from above.

For showing that, one notices that the hyperplane $H$ defined by $\left\{z \in \mathbb{R}^{r} \mid u^{\prime} z=0\right\}$ contains $z$ and that $\left\{z \in H \mid w+z \in \mathbb{R}_{++}^{r}\right\}$ is bounded from above.

Before showing that the converse property is true, let us define the typical consumer's problem, given Al. Given $Z \in G_{r, n}$, choose $z$ in order 
to maximize $\tilde{u}(w+z)$ subject to $z \in Z$. Consider the set $\{z \in z \mid \tilde{u}(w+z) \geq \tilde{u}(w)\}$. This set is compact iff the set $\left\{z \in z \mid w+z \in \mathbb{R}_{++}^{r}\right\}$ is bounded from above. Choose a $P$ which induces $z$. Then the typical consumer's maximization problem becomes

$$
\operatorname{Max} \tilde{u}(w+z)
$$

subject to $P^{\prime} z=0$.

A necessary and sufficient condition for the existence of a solution is that there exists $b \in \mathbb{R}^{r-n}$ such that $P b=u(w+z)$ for some $z \in z$. Thus, if $Z^{\perp} \cap \mathbb{I R}_{++}^{r}$ is empty, the typical consumer's maximization problem has no solution and this means, as $\tilde{u}($ ) is continuous, that the set $\{z \in z \mid \tilde{u}(w+z) \geq \tilde{u}(w)\}$ which is not empty $(0 \in z)$ and closed, is not bounded.

Thus it is natural to restrict the analysis to the case of $z \in \tilde{G}_{r, n}$. Given $\mathrm{Al}$, the solution to the consumer's problem is unique. Let $z=\xi(z)$ be this solution, for each $z \in \tilde{G}_{r, n}$.

Proposition 1: Given Al, consider a sequence $\left\{z^{\nu}\right\} \subset G_{r, n}$ converging towards $z^{0}$ with $z^{\nu} \in \tilde{G}_{r, n} \quad \forall v$ and $z^{0} \notin \tilde{G}_{r, n}$. Then $z^{\nu}=\xi\left(z^{\nu}\right)$ is such that $\left\|z^{v}\right\|+\infty$.

Proof: Write the consumer's problem in the following way. Max $\tilde{u}(x)$ subject to $x \in z_{w}^{\nu}$ where $z_{w}^{\nu}$ is the n-dimensional linear manifold parallel to $z^{\nu}$ such that $w \in z_{w^{*}}^{\nu}$. Recall that $w_{h}>0$ th. Consider the sequence $\left\{x^{\nu}\right\}$ of unique solutions. Ad absurdum, assume that $\left\|x^{v}\right\|$ does not go to infinity. 
Without loss of generality, one can assume then that the sequence $\left\{x^{\nu}\right\}$ converges towards $x^{0}$. Clearly, $x^{0} \in z_{w}^{0}$ (closed graph property). Moreover as $w \gg 0$, for each $\bar{x} \in z_{w}^{0} \cap \mathbb{R}_{++}^{r}$ and for each $\varepsilon>0$ one can find $\nu$ and $\vec{x} \in Z_{w}^{\nu} \cap \mathbb{R}_{++}^{r}$ such that $\left\|\bar{x}^{\nu}-\bar{x}\right\|<\varepsilon$ (lower hemicontinuity). It follows that $x^{0}$ maximizes $\tilde{u}(x)$ in $z_{w}^{0}$ : a contradiction. Q.E.D.

Remark: The proof of Proposition 1 will be, maybe, clearer if one argues in the following way. For each $Z$, one can find a regular $r \times n$ matrix $T$ such that $z=\left\{z \in \mathbb{R}^{\mathrm{r}} \mid \exists a \in \mathbb{R}^{\mathrm{n}}\right.$ such that $\left.\mathrm{T} a=z\right\}$. Consider the class of regular matrices $\eta^{T}$ which has as independent rows the rows $\left\{h_{1}<\ldots<h_{j}<\ldots<h_{n}\right\}$ and such that the $n \times n$ submatrix formed by these rows is the unitary $n \times n$ matrix. As $\left\{w_{n}\right\}_{n}$ is an open cover of $G_{r, n}, z^{0} \in W_{\bar{n}}$ for some $\bar{n}$. Without loss of generality, one can assume that $z^{\nu} \in W_{n}$ for each $\nu$. Then $\left\{z^{\nu}\right\}+z^{0}$ means that $n^{-T^{\nu}}+n^{-T^{0}}$. It is then clear that if $z^{\nu}=\bar{T}^{\nu} a^{\nu}$ and $\left\{z^{\nu}\right\} \rightarrow z^{0}$ with $\left\{T^{\nu}\right\} \rightarrow T^{0}$, then $\exists a^{0}$ such that $z^{0}=\bar{T}^{0} a^{\bar{\gamma}}$. Actually $a_{1}^{\nu}=z_{h_{1}}^{\nu}, \cdots, a_{n}^{\nu}=z_{h_{n}}^{\nu} \quad$ and as $\left\{z^{\nu}\right\}+z^{0}$, then $\left\{a^{\nu}\right\}+a^{0}$. For showing the property of "lower hemi-continuity", one argues in the following way. Consider the $a^{0}$ such that $\bar{n}^{0} a^{0}=z^{0}$ with $w+z^{0} \gg 0$. Then the sequence of $\bar{n}^{-T^{\nu}} a^{0}=z^{\nu}$ will converge towards $z^{0}$ and for $v$ large enough $w+z^{\nu} \gg 0$.

Consider some $Z \in \tilde{G}_{r, n}$. Choose a $P$ which induces $Z$. Choose moreover $\gamma$ such that the rows of the ordered set $\left\{h_{1}, \ldots, h_{j}, \ldots, h_{r-n}\right\}$ are linearly independent. For reference, one shall write often $\gamma^{P}$. When $Z$ varies in $W_{\gamma}$, one can write $z=\xi_{\gamma}\left({ }_{\gamma} P\right)$. For ease of notation, one shall often write $z=\xi(P)$.

The first order conditions which characterize the solution of the 
consumer's problem can then be written (where $U \equiv U_{x x}=\left[d^{2} u / d x^{2}\right]$ and $u \equiv[\tilde{d u} / d x]):$

$$
\begin{aligned}
& u-P b=0 \text { with } b \in \mathbb{R}^{r-n} \\
& P^{\prime} z=0 .
\end{aligned}
$$

Notice that if $\gamma^{P}$ is normalized, we have $b^{j}=u_{h_{j}} j=1, \ldots, r-n$.

Lemma 1: Under $A 1$, if $z=\xi(P)$, the bordered Hessian matrix is non singular:

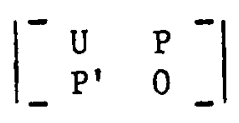

where $U$ is evaluated at $w+z$.

Proof: It is known that Al implies that $\left|\begin{array}{cc}U_{u} & u^{\prime} \\ u^{\prime} & 0\end{array}\right|$ is non singular. As $\mathrm{u}=\mathrm{Pb}$, one gets the result.

Keeping $w$ constant, let us differentiate totally the system of first order conditions.

$$
\begin{aligned}
& U d z-B d P-P d b=0 \\
& P^{\prime} d z+\overline{z d P}=0
\end{aligned}
$$

In this system $B=\left[B^{1}, ., B^{j}, ., B^{r-n}\right]$ where $B^{j}$ is the $r \times r$ matrix 


$$
\left|\begin{array}{lll}
b^{j} & & \\
& \ddots & \\
& & b^{j}
\end{array}\right|
$$

$d P$ is the $(r-n) \cdot r$ column vector $\left[d p^{1}, \ldots, d p^{r-n}\right]$ for which $d p_{h_{1}}^{j}=0, \ldots, d p_{h}^{j}=0$ for each $j$. and $\bar{z}$ is the $(r-n) \times(r-n) \cdot r$ matrix

$$
\left|\begin{array}{cc}
{\left[z_{1}, \ldots, z_{r}\right]} & 0 \\
0 & \ddots\left[z_{1}, \ldots, z_{r}\right]
\end{array}\right|
$$

Lemma 2: Given $A 1$, the function $\xi(P)$ is differentiable.

Proof: Choose an admissible $\gamma$. The Jacobian matrix of $\xi$ with respect to $[z, b]$ is equal to

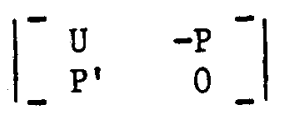

As this matrix is nonsingular, it is possible to apply the implicit function theorem to the system of first order conditions. Q.E.D.

In order to compute the counterpart of the usual slutsky matrix, one shall argue in the following way.

The implicit function theorem implies that

$$
\left|\begin{array}{c}
D z / d P \\
-d b / d P
\end{array}\right|=\left|\begin{array}{cc}
U & P^{-} \\
P^{\prime} & 0
\end{array}\right|^{-1}\left|\begin{array}{|}
\frac{B}{z}
\end{array}\right|
$$

Write 


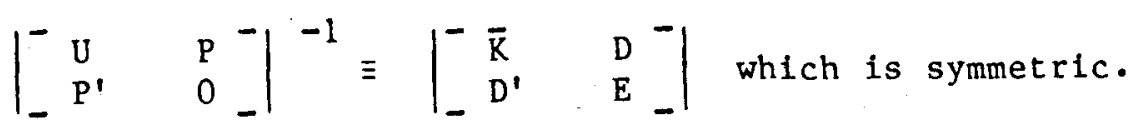

where $D$ is $r \times(r-n)$ and $E$ is $(r-n) \times(r-n)$.

Then
a) $\mathrm{U} \cdot \overline{\mathrm{K}}+\mathrm{PD}^{\prime}=\mathrm{I}_{\mathrm{r}}$ (the unitary $\mathrm{r} \times \mathrm{r}$ matrix)
b) $\mathrm{U} \cdot \mathrm{D}+\mathrm{PE}=0$
c) $P^{\prime} \overline{\mathrm{K}}=0$
d) $P^{\prime} D=I_{(r-n)}$ (the unitary $(r-n) \times(r-n)$ matrix).

Thus $\frac{d z}{d P}=\bar{K} B+D \bar{z}$. Obviously $\bar{K} B$ is the matrix of substitution effects and $\bar{D} \bar{z}$ is the matrix of income effects. $\overline{\mathrm{K}} B$ can be named the matrix of substitution effects because $\frac{\mathrm{d} z}{\mathrm{dP}}=\overrightarrow{\mathrm{K} B}$ when $\bar{z}=0$ i.e. when one considers a no trade allocation. Consider first the matrix of income effects: $\bar{D} \bar{z}$. As $P^{\prime} D=I(r-n)$, each column $\left(d^{j}\right)$ of $D$ can be considered as the deviations of $z$ implied by a variation of $v^{j}$ in the equation $P^{\prime} z=v$, for $j=1, \ldots, r-n . \quad \bar{K} B$ is a $r \times r(r-n)$ matrix

$$
\overline{\mathrm{K}} B=\left[\overline{\mathrm{K}} B{ }^{1}, \ldots, \overline{\mathrm{K} B}{ }^{(\mathrm{r}-\mathrm{n})}\right]=\left[\overline{\mathrm{K}} \cdot \mathrm{b}^{1}, \ldots, \overline{\mathrm{K}} \cdot \mathrm{b}^{\mathrm{r}-\mathrm{n}}\right]
$$

One wants to show that $\overline{\mathrm{K}}$ has rank $\mathrm{n}$, for each $\mathrm{j}$. Obviously as $P^{\prime} \bar{K}=\bar{K} \cdot P=0, \bar{K}$ has rank at most $r-(r-n)=n$ because the $(r-n)$ column vectors of $P$ are independent. As $U \bar{K}=I_{r}-P \cdot D^{\prime}$, and $I_{r}-P D^{\prime}$ is a matrix of rank $n$ (because $P \cdot D^{\prime}$ has rank $(r-n)$ ) the rank of $\bar{k}$ is at least n. Then it is exactly n.

Finally, let us show that $\overline{\mathrm{K}}$ is negative semi definite and more precisely that $y^{\prime} \bar{x} y<0$ for each $y \neq 0$ which does not belong to the subspace generated by P. Premultiplication of (a) by $\bar{K}$ gives 
$\overline{\mathrm{K}} \cdot \mathrm{U} \cdot \overline{\mathrm{K}}=\overline{\mathrm{K}} \mathrm{PD} \mathrm{D}^{\prime}=\overline{\mathrm{K}}$ or $\overline{\mathrm{K}} \mathrm{U} \overline{\mathrm{K}}=\overline{\mathrm{K}}$ because $\overline{\mathrm{K}} \cdot \mathrm{P}=\mathrm{P}^{\prime} \overline{\mathrm{K}}=0(\mathrm{c})$. Thus $y^{\prime} \bar{K} y=y^{\prime} \bar{K} U \bar{K} y$. Let $q=\bar{k} y$. Note that $p^{\prime} q=p^{\prime} \bar{K} y=0$. Thus $u^{\prime} \cdot q=0$. Thus if $q \neq 0 \quad y^{\prime} \bar{K} y=q^{\prime} \cup q<0$. The case $q=0$ occurs when $y=P b$ for some $b \in \mathbb{R}^{r-n}$. It is not difficult to understand why $\bar{k}$ has rank $n$ and more precisely why $y^{\prime} \bar{k} y<0$ for each $y$ which does not belong to the $(r-n)$ dimensional subspace generated by $p$. Given $\gamma$, the prices $p_{h}^{j}$ for $h=h_{1}, \ldots, h=h_{(r-n)}$ are kept fixed.

It follows that for each $y$ with $y_{h}=0$ for $h=h_{1}, \ldots, h=h_{(r-n)}, \bar{k} y \neq 0$ and $y \bar{k} y^{\prime}<0$ because then there does not exist $b$ such that $y=P b \neq 0$.

Proposition 2: Consider the demand function $z \in \xi(Z)$ for $z \in \tilde{G}_{I, n}$. Under Al it is differentiable. For each admissible $\gamma$ which concerns the set of goods $\left\{h_{1}, \ldots, h_{j}, \ldots, h_{r-n}\right\}$ :

$$
\frac{d z}{d_{\gamma} P}=\left[\gamma k \cdot b^{1}, \cdots \cdot{ }_{\gamma} k \cdot b^{r-n}\right]+{ }_{\gamma} D \bar{z}
$$

where $\gamma^{K}$ is negative semi definite with $\gamma^{K \cdot y}=0$ for each $y$ which belongs to the $(r-n)$ dimensional subspace generated by $\gamma^{P}$, and where $\left[b^{1}, \ldots, b^{r-\dot{n}}\right]=b$ is defined uniquely by $u=\gamma^{P} \cdot b$. In the case where $\gamma^{P}$ is normalized ( $\gamma$ defines the ordered set $\left.\left(h_{1}, \ldots, h_{j}, \ldots, h_{r-n}\right\}\right)$, then $b^{j}=u_{h_{j}}>0$.

Finally, one has to find the counterpart of Walras law. Consider $m$ agents $i=1, \ldots, m$. After having an admissible $\gamma$ (concerning goods $\left.h_{1}, \ldots, h_{r-n}\right)$ one writes $\sum_{i} \xi_{i h}(P)=0 k=1, \ldots, r$. However, as $P^{\prime} \xi_{i}(P)=0$ for each $i$, one can conclude that if $\sum_{i} \xi_{\text {ih }}(P)=0$ for $h \neq h_{1}, \ldots, h \neq h_{r-n}$. then $\sum_{i} \xi_{i h}(P)=0$ for $h=1, \ldots, r$. Let us write $\hat{\xi}_{i}(P)$ the demand 
function for goods $h \neq h_{j}, j=1, \ldots, r-h$. Let us now introduce $w_{i}$ in the analysis and write $z_{1}=\xi_{1}\left(P, w_{1}\right)$. Consider the system

$$
\begin{aligned}
u_{i}\left(w_{i}+z_{i}\right)-P b_{i} & =0 \cdot \text { with } b_{1} \in \mathbb{R}^{r-n} \\
P^{\prime} z_{i} & =0 .
\end{aligned}
$$

Keeping $P$ constant, one gets by differentiation

$$
\begin{aligned}
U_{i} d z_{i}+U_{i} d w_{1}-P d b_{i} & =0 \\
P^{\prime} d z_{i} & =0
\end{aligned}
$$

One gets

$$
\left|\begin{array}{c}
\mathrm{dz}_{i} / \mathrm{dw}_{i} \\
\mathrm{db}_{1} / \mathrm{dw}_{i}
\end{array}\right|=\left|\begin{array}{cc}
\overline{\mathrm{K}} & \mathrm{D}_{i} \\
\mathrm{D}_{1}^{\prime} & \mathrm{E}_{i}
\end{array}\right|\left|\begin{array}{c}
\mathrm{u}_{i} \\
0
\end{array}\right|
$$

Thus $\left|\frac{\mathrm{dz}_{i}}{\mathrm{dw}_{i}}\right|=\overrightarrow{\mathrm{k}}_{i} \mathrm{U}_{i}=\mathrm{U}_{i} \overline{\mathrm{k}}_{1}$

One has seen that the submatrix of $U_{i} \bar{K}_{i}$ formed with rows $h \neq h_{j}, j=1, \ldots, r-n$ has rank $n$. Thus, let $w=\left[w_{1}, \ldots, w_{m}\right]$ :

$$
\left|-\frac{d \Sigma_{\gamma} \hat{\xi}_{i}\left({ }_{\gamma} P, w_{i}\right)}{d w}\right|
$$

has rank n. 
Proposition 3: Consider $\sum \xi_{i}\left(Z, w_{i}\right)$. For each admissible $\gamma$, let be $\sum_{i} \hat{\xi}_{i}\left({ }_{\gamma} P, w_{i}\right)$ the aggregate excess demand function for all goods except the $(r-n)$ goods concerned by $\gamma$. Then

$$
\left|-\frac{d \sum \gamma_{1}\left(\gamma p, w_{1}\right)}{d w}\right|
$$

has rank $n$, and $\sum_{i} \hat{\xi}_{i}\left({ }_{\gamma} P, w\right)=0 \Rightarrow \sum_{i} \gamma_{i} \xi_{\gamma}\left({ }^{P}, w_{i}\right)=0$.

Remark: Consider now, for each $n(n \leq r-1)$ the set

$$
\vec{\Gamma}=\left\{(w, Z)\left|w \in \Omega, Z \in \tilde{G}_{r, n}\right| \sum \xi_{i}\left(Z, w_{i}\right)=0\right\}
$$

Using the argument developed in the next part, it is straightforward to show that $\bar{\Gamma}$ is a smooth manifold of dimension $(m \cdot r)+n(r-n)-n$.

When $n=r-1$, it is a manifold of dimension $m^{\bullet} r$. This is obviously related to the fact that all $(r-1)$ quasi-regular market structures are weakly equivalent. 


\section{Footnotes}

* I thank B. Allen, Y. Balasko, D. Cass, M. Jerison, B. Jun and P. Siconolfi for their advice.

1. See Y. Balasko and D. Cass (1985) for a somewhat different approach.

2. See Y. Younés (1985) and (1984). 


\section{References}

Y. Balasko and D. Cass (1985), "Regular Demand with Several, General Budgetary Constraints", Caress Working Paper, University of Pennsylvania.

Y. Balasko and D. Cass (1986), "The Structure of Financial Equilibrium I."

D. Cass (1986), "Competitive Equilibrium with Incomplete Financial Markets," Caress Working, University of Pennsylvania.

S. Chae (1985), "Existence of Competitive Equilibrium with Incomplete Markets," Working Paper, Rice University.

J. Dieudonné (1970), Elements d'analyse. 3, Gauthier-Villars.

D. Duffie and W. Shafer (1985), "Equilibrium in Incomplete Markets I", Working Paper, University of California at Berkeley.

D. Gale (1982), Money: In Equilibrium. Cambridge Economic Handbooks.

J. Geneakoplos and A. MasColel1 (1985), "Real Indeterminacy with Financial Assets," Cowles Foundation Discussion Paper.

0. Hart (1975) "On the Optimality of Equilibrium when the Market Structure is Incomplete," Journal of Economic Theory, 11 p. 418-443.

M. Magill and W. Shafer (1985), "Equilibrium and Efficiency on a Canonical Asset Trading Mode1", Working Paper, University of Southern California.

D. McManus (1984), "Generic Existence of Equilibrium and Optimality Properties in an Economy with Future Markets", Working Paper, University of Pennsylvania.

H. Polemarchakis (1986), "Equilibrium with Incomplete Markets: A Simple Proof and Further Results", Working Paper.

R. Radner (1972), "Existence of Equilibrium Plans, Price and Price Expectation In a Sequence of Markets", Econometrica, 40 p. 289-303.

R. Repullo (1983), "Equilibrium and Efficiency in Economies with a Sequence of Markets", Chapter 6, Ph.D. Dissertation, London School of Economics.

J. Werner (1985), "Equilibrium on Economies with Incomplete Financial Markets", Journal of Economic Theory 36, p. 110-119.

Y. Younés (1984), "General Competitive Equilibrium with Asymmetric Information and Signalling", CARESS Working Paper, University of Pennsylvania

Y. Younes (1985), "On the Theory of Incomplete Markets", Caress Working Paper, University of Pennsylvania. 


\section{ANNEX}

The objective of this annex is to emphasize that we did not choose the most general formulation of a market structure, even when Al is made. Let be $M=\left[E^{1}, \ldots, E^{B}, \ldots, E^{N}\right]$ a market structure where $E^{\beta}$ is a vector subspace of $\mathbb{R}^{r}$. Each agent 1 choose a $z_{1}^{\beta} \in E^{\beta}$.

Define $\tilde{z}_{1}=\left[z_{1}^{1}, \ldots, z_{1}^{B}, \ldots, z_{1}^{N}\right]$.

$\tilde{z}_{i}$ is possible for $i$ if

(i) $z_{i}^{\beta} \in E^{\beta}$ HB.

(ii) $w_{i}+\sum_{\beta} z_{i}^{\beta} \in x_{i}$.

$\tilde{z}=\left[\tilde{z}_{1}, \ldots, \tilde{z}_{\mathrm{m}}\right]$ is balanced if $\sum_{i} z_{i}^{\beta}=0 \quad \#$.

$\left(\tilde{z}^{*}, p^{*}\right)$ is an equilibrium if

(i) $\mathrm{p}^{*} \in \mathrm{s}^{\mathrm{r}-1}$

(ii) $\tilde{z}^{*}$ is balanced

(iii) $\forall i: \tilde{z}_{i}^{*}$ maximizes $\tilde{u}_{i}\left(w_{i}+\sum_{\beta} z_{i}^{\beta}\right)$

in the set $\left\{\tilde{z} \mid z_{i}^{\beta} \in E^{\beta} \forall \beta, w_{i}+\sum_{\beta} z_{i}^{\beta} \in X_{i}\right.$ and $\left.p^{*} z_{i}^{\beta}=0 \forall \beta\right\}$.

The following examples show that, even when $\mathrm{Al}$ is made, it is not always possible to decompose a market $\mathrm{E}^{\beta}$ in simple markets.

Example: $\mathbf{r}=3$ and consider a market $E$ defined by

$$
E=\left\{z \in \mathbb{R}^{3} \mid z_{2}=-2 z_{3}\right\}
$$

$A$ basis for $E$ is

$$
\left|\begin{array}{rr}
1 & 0 \\
0 & 2 \\
0 & -1
\end{array}\right|
$$


For $p \in \stackrel{O}{S}^{r-1}$ such that $p_{3} \neq 2 p_{2}$, good 1 can be traded. For $p_{3}=2 p_{2}$, one has necessarily $z_{1}=0$. E is not a market on which a finite number of disjunct bundles of goods are exchanged.

Example: $\quad r=3$ and a market $E$ is defined through the basis

$$
\left|\begin{array}{cc}
1 & 1 \\
-1 / 2 & -2 \\
0 & 1
\end{array}\right|
$$

It can be defined also by the relation

$$
z_{1}+2 z_{2}+3 z_{3}=0
$$

Thus if $p$ is not colinear with the vector $\{1,2,3\}^{\prime}$, the set

$$
\left\{z \in E \mid p^{\prime} \cdot z=0\right\}
$$

has dimension 1 . When $p=\{1,2,3\}^{\prime}$, it has dimension 2 . One get thus an increase of rank, and not a decrease, at some critical points. $E$ is not a market on which a finite number of disjunct bundles of goods are exchanged. Obviously, the two examples are different. In the first one, there is a bundle composed of good 1 alone which can be exchanged against a "bundle" containing good 3 in "negative proportions". In the second one, this is not the case and one can get increases of rank.

Let us deal with the first case. One assumes A2': $M=\left[E^{1}, \ldots, E^{\beta}, \ldots, E^{N}\right]$ is such that for each $B$, one can find a basis $\left[e_{1}^{\beta}, \ldots, e_{k}^{\beta}, \ldots, e_{n(\beta)}^{\beta}\right]$. for each $E^{\beta}$ such that $e_{n(\beta)}^{\beta}$ does not contain negative elements. 
One chooses the bundle $e_{n(B)}^{\beta}$ as the "numeriare". Define now a "bundle" as a point in $\mathbb{R}^{r} \backslash\{0\}$. Thus there are $n(B)$ "bundles" of goods in market $\beta$. Then, under $A 1$, one can construct, for each $\beta,[n(\beta)-1]$ simple markets by assuming that the last bundle is exchanged for the first $[n(B)-1]$ "bundles" . One gets $[n(\beta)-1]$ "simple" markets $\alpha$ for each $\beta$. Let be $n \equiv N[n(\beta)-1]$. One formulates still a market structure by $\Lambda=\left[\Lambda^{+}, \Lambda^{-}\right]$. Notice that for each $\alpha, \lambda^{\alpha-}$ belongs to $\mathbb{R}_{+}^{r} \backslash\{0\}$, because for each $\beta, e_{n(\beta)}^{\beta} \in \mathbb{R}_{+}^{r} \backslash\{0\}$. Let be $\pi=\left[\pi^{1}, \ldots, \pi^{\alpha}, \ldots, \pi^{n}\right]$ with $\pi^{\alpha} \in \mathbb{R} \forall \alpha$. Thus

$$
\tilde{T}(\pi)=\Lambda^{+}-\Lambda^{-}[\pi]
$$

and with each $p \in \mathbb{R}_{++}^{r}$, one associates one $\pi$ by the relation

$$
\left.\pi^{\alpha}=F_{\Lambda}^{\alpha}(p)=\sum_{h} p_{h} \lambda_{h}^{\alpha+} \cdot \underset{h}{\left[\sum\right.} p_{h} \lambda_{h}^{\alpha-}\right]^{-1} * \alpha
$$

Thus $T(p) \equiv \tilde{T}\left[F_{\Lambda}(\pi)\right]$ is a smooth function of $p \in \mathbb{R}_{++}^{r}$.

Definition: Given $\Lambda$ and $w$, an equilibrium is a couple $\left[\pi^{*}, z^{*}\right] \in \mathbb{R}^{\mathrm{n}} \times \tilde{G}_{\mathrm{r}, \mathrm{n}}$ such that

(i) $z^{*}$ is the subspace generated by $\tilde{\mathrm{T}}\left(\pi^{*}\right)$

(ii) $\sum_{i} \xi_{i}\left(z^{*}, w_{i}\right)=0$.

For defining a pseudo equilibrium, we replace (ii) by: $\mathrm{z}^{*}$ contains the subspace generated by $\tilde{T}\left(\pi^{*}\right)$. Now, notice that as $[\tilde{T}(\pi)]^{\prime} u_{i}=0 \forall i, \pi$ will remain bonded at a (pseudo-) equilibrium when $w$ varies in a compact set of $\boldsymbol{U}$ ( see Chapter B). 
Remark: When $\mathrm{A}^{\prime}$ ' is not made, our device for choosing a "bundle" as "numeraire" leads to the conclusion that $\mathrm{F}_{\Lambda}^{\alpha}(\mathrm{p})$ is no more a function, for each $\alpha$. For some $p \in \mathbb{R}_{++}^{r}$, one may get $\sum_{h} p_{h} \lambda_{h}^{\alpha+}=0$ and $\sum_{h} p_{h} \lambda_{h}^{\alpha-}=0$. In the second example, one gets for $p=[1,2,3]^{\prime}, p^{\prime} e_{1}=p^{\prime} e_{2}=0$ and there is an increase of rank. The analysis of the situation where A2' is not made is beyond the scope of this paper. 